Article

\title{
Eccentricity-Based Topological Indices of a Cyclic Octahedron Structure
}

\author{
Manzoor Ahmed Zahid ${ }^{1}$, Abdul Qudair Baig ${ }^{2}$ and Muhammad Naeem ${ }^{2, *}$ \\ and Muhammad Razwan Azhar ${ }^{3}$ (D) \\ 1 Department of Mathematics, COMSATS University Islamabad, Sahiwal 57000, Pakistan; \\ manzoor_zahid@hotmail.com \\ 2 Department of Mathematics, The University of Lahore, Pakpattan Campus, Pakpattan 57400, Pakistan; \\ aqbaig1@gmail.com \\ 3 Department of Mathematics, Govt. High School Burhan, Attock 43600, Pakistan; \\ razwanazhar2015@gmail.com \\ * Correspondence: naeempkn@gmail.com
}

Received: 10 July 2018; Accepted: 9 August 2018; Published: 17 August 2018

\begin{abstract}
In this article, we study the chemical graph of a cyclic octahedron structure of dimension $n$ and compute the eccentric connectivity polynomial, the eccentric connectivity index, the total eccentricity, the average eccentricity, the first Zagreb index, the second Zagreb index, the third Zagreb index, the atom bond connectivity index and the geometric arithmetic index of the cyclic octahedron structure. Furthermore, we give the analytically closed formulas of these indices which are helpful for studying the underlying topologies.
\end{abstract}

Keywords: molecular graph; eccentric connectivity polynomial; eccentric connectivity index; total eccentricity; average eccentricity; first Zagreb index; second Zagreb index; third Zagreb index; atom bond connectivity index; geometric arithmetic index; cyclic Octahedron structure

JEL Classification: 05C12; 05C90

\section{Introduction}

Graph theory has advanced greatly in the field of mathematical chemistry. Chemical graph theory has become very popular among researchers because of its wide application in mathematical chemistry. The molecular topological descriptors are the numerical invariants of a molecular graph and are very useful for predicting their bioactivity. A great variety of such indices have been studied and used in theoretical chemistry, by pharmaceutical researchers, in drugs, and in other different fields. There is considerable usage of graph theory in chemistry. Chemical graph theory is the topology branch of mathematical chemistry which applies graph theory to the mathematical modeling of chemical occurrence. A lot of research has been done in this area in the last few decades. This theory has a major role in the field of chemical sciences.

In reference [1,2], W. Gao et al. computed the electron energy of molecular structures through the forgotten topological index. Also, they computed the generalized atom bond connectivity index of several chemical molecular graphs. In reference [3-5], the authors studied topological indices of networks and nanotubes. The topological index of aztec diamonds was discussed in reference [6] by M. Imran et al. Some degree-based and eccentricity-based topological indices of oxide networks and tetra sheets were described in reference [7-9] by A. Q. Baig et al., respectively.

Recently the eccentric atom bond connectivity index of linear polycene parallelogram benzenoid was introduced by reference [10]. Sierpinski graphs constitute an extensively studied family of 
graphs of fractal nature and have been applied in topology, the mathematics of the Tower of Hanoi, computer science, and elsewhere [11]. The Sierpinski graphs were introduced in reference [12] by Klavzar and Milutinovic. The average eccentricity and standard deviation for all Sierpiński graphs $\left(S_{p}^{n}\right)$ was established by reference [13]. The extremal properties of the average eccentricity as well as the conjectures and autographics were obtained by reference [14], in which the AutoGraphiX(AGX) computer system was developed by the GERAD group from Montreal [15-17]. AGX is an interactive software designed to help find conjectures in graph theory. The bounds on the mean eccentricity of graph and also the change in mean eccentricity when a graph is replaced by a subgraph was established by reference [18]. For trees with a fixed diameter, fixed matching number and fixed number of pendent vertices, the lower and upper bounds of average eccentricity were found by reference [19].

An undirected graph is a pair $(G=(V, E))$, where $V$ is the set of vertices, and $E \subseteq\left(\begin{array}{l}V \\ 2\end{array}\right)$ is a set of edges. In molecular graph theory, the vertices represent atoms, and the edges represent bonds between the atoms.

If $u, v \in V$, then the distance $(d(u, v))$ between $u$ and $v$ is defined as the length of any shortest path in $G$ connecting $u$ and $v$. We denote $d_{v}$ as the number of edges incident to vertex $v$ in $G$. The eccentricity of $u$ is the distance of vertex $u$ from the farthest vertex in $G$. In mathematical form, this is shown as $\varepsilon(u)=\max \{d(u, v) \mid \forall v \in V\}$. Table 1 describes the eccentricity-based indices and polynomials which have been introduced over the years.

Table 1. Eccentric-based indices.

\begin{tabular}{ccccc}
\hline S.No. & Introduced by & Index Name & Notation & Formula \\
\hline 1 & V. Sharma et al. [20] & Eccentric connectivity index & $\xi(G)$ & $\sum_{v \in V} d_{v} \varepsilon(v)$ \\
\hline 2 & M. Alaeiyan et al. [21,22] & Eccentric connectivity polynomial & $E C P(G, x)$ & $\sum_{v \in V} d_{v} x^{\varepsilon(v)}$ \\
\hline 3 & Farooq et al. [23] & Total eccentricity index & $\zeta(G)$ & $\sum_{v \in V} \varepsilon(v)$ \\
\hline 4 & F. Bukley et al. [24] & Average eccentricity & avec(G) & $\frac{1}{n_{v \in V} \varepsilon(v)}$ \\
\hline 5 & D. Vukičević et al. & First Zagreb eccentric index & $M_{1}^{*}(G)$ & $\sum_{u v \in E}[\varepsilon(u)+\varepsilon(v)]$ \\
\hline 6 & G. Ghorbani et al. [25,26] & Second Zagreb eccentric index & $M_{1}^{* *}(G)$ & $\sum_{v \in V}[\varepsilon(v)]^{2}$ \\
\hline 7 & third Zagreb eccentric index & $M_{2}^{*}(G)$ & $\sum_{u v \in E} \varepsilon(u) \varepsilon(v)$ \\
\hline
\end{tabular}

The aim of this paper is to compute and compare the above described eccentric-based topological indices for a cyclic octahedron structure of dimension $n$.

\section{Main Results and Discussion}

In this section, we discuss the cyclic octahedron structure and give closed formulae of certain topological indices for this network. Here, we find the analytically closed results of the eccentric connectivity polynomial, the eccentric connectivity index, the total eccentricity index, the average eccentricity index, and the eccentricity-based geometric-arithmetic and atom bond connectivity indices for the cyclic octahedron structure.

An octahedron graph, as shown in Figure 1, is a polyhedral graph corresponding to the skeleton of the octahedron, one of the five Platonic solids. This Platonic graph consists of six vertices and 12 
edges. The analogs of this structure play vital roles in the field of reticular chemistry, which deals with the synthesis and properties of metal-organic frameworks [11,29].

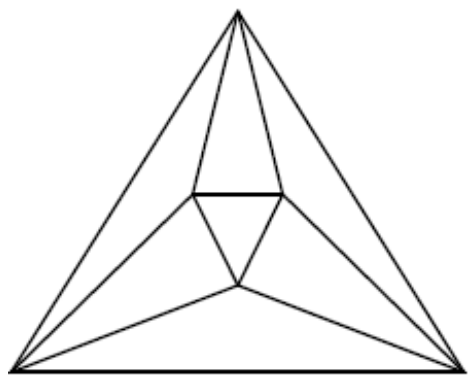

Figure 1. Structure of an octahedron.

The different types of octahedral structures arise from the ways that these octahedra can be connected. The cyclic octahedral structure of dimension $n$ is denoted by $C Y O_{n}$, and it is obtained by arranging $n$ octahedra in cyclic order, as shown in Figure 2. For $n \geq 3, C Y O_{n}$ consists of $5 n$ vertices and $12 n$ edges. To compute the said indices and polynomials, we partitioned the vertices and edges of $\mathrm{CYO}_{n}$ in certain ways in Tables 2-7. To understand the tables and the partitions that they describe, we give detailed captions of each table. We computed the exact formulas for the above mentioned topological indices of the cyclic octahedral structure as follows.

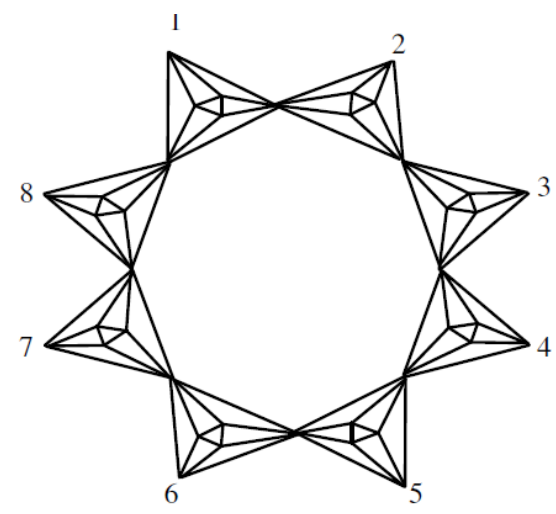

Figure 2. Cyclic octahedral structure $\left(\mathrm{CYO}_{8}\right)$.

Table 2. Vertex partition of the cyclic octahedron structure for ( $n$-levels) where $n$ is odd based on the degree and eccentricity of each vertex with the existence of their frequencies.

\begin{tabular}{cccc}
\hline$d_{u}$ & $\varepsilon(u)$ & Frequency & Range of $\boldsymbol{n}$ \\
\hline 4 & $\frac{n+3}{2}$ & $4 n$ & $n \geq 3$ \\
8 & $\frac{n+1}{2}$ & $n$ & $n \geq 3$ \\
\hline
\end{tabular}

Table 3. Vertex partition of the cyclic octahedron structure for ( $n$-levels) where $n$ is even based on the degree and eccentricity of each vertex with the existence of their frequencies.

\begin{tabular}{cccc}
\hline$d_{\boldsymbol{u}}$ & $\boldsymbol{\varepsilon}(\boldsymbol{u})$ & Frequency & Range of $\boldsymbol{n}$ \\
\hline 4 & $\frac{n+2}{2}$ & $2 n$ & $n \geq 4$ \\
4 & $\frac{n+4}{2}$ & $2 n$ & $n \geq 4$ \\
8 & $\frac{n+2}{2}$ & $n$ & $n \geq 4$ \\
\hline
\end{tabular}


Table 4. Vertex partition of the cyclic octahedron structure for ( $n$-levels) where $n$ is odd based on the eccentricity of each vertex with the existence of their frequencies.

\begin{tabular}{ccc}
\hline$\varepsilon(u)$ & Frequency & Range of $\boldsymbol{n}$ \\
\hline$\frac{n+1}{2}$ & $n$ & $n \geq 3$ \\
$\frac{n+3}{2}$ & $4 n$ & $n \geq 3$ \\
\hline
\end{tabular}

Table 5. Vertex partition of the cyclic octahedron structure for ( $n$-levels) where $n$ is even based on the eccentricity of each vertex with existence of their frequencies.

\begin{tabular}{ccc}
\hline$\varepsilon(u)$ & Frequency & Range of $\boldsymbol{n}$ \\
\hline$\frac{n+2}{2}$ & $3 n$ & $n \geq 4$ \\
$\frac{n+4}{2}$ & $2 n$ & $n \geq 4$ \\
\hline
\end{tabular}

Table 6. Edge partition of the cyclic octahedron structure for ( $n$-levels) where $n$ is odd based on the eccentricity of end vertices with the existence of their frequencies.

\begin{tabular}{ccc}
\hline$(\varepsilon(u), \varepsilon(v))$ & Frequency & Range of $\boldsymbol{n}$ \\
\hline$\left(\frac{n+1}{2}, \frac{n+1}{2}\right)$ & $n$ & $n \geq 3$ \\
$\left(\frac{n+1}{2}, \frac{n+3}{2}\right)$ & $6 n$ & $n \geq 3$ \\
$\left(\frac{n+3}{2}, \frac{n+3}{2}\right)$ & $5 n$ & $n \geq 3$ \\
\hline
\end{tabular}

Table 7. Edge partition of the cyclic octahedron structure for ( $n$-levels) where $n$ is even, based on the eccentricity of end vertices with the existence of their frequencies.

\begin{tabular}{ccc}
\hline$(\varepsilon(u), \varepsilon(v))$ & Frequency & Range of $n$ \\
\hline$\left(\frac{n+2}{2}, \frac{n+2}{2}\right)$ & $5 n$ & $n \geq 4$ \\
$\left(\frac{n+2}{2}, \frac{n+4}{2}\right)$ & $6 n$ & $n \geq 4$ \\
$\left(\frac{n+4}{2}, \frac{n+4}{2}\right)$ & $n$ & $n \geq 4$ \\
\hline
\end{tabular}

\subsection{Eccentric Connectivity Polynomial}

Then, using the following theorems, we computed the eccentric polynomial of the cyclic octahedron structure $\left(\operatorname{ECP}\left(\mathrm{CYO}_{n}, x\right)\right)$.

Theorem 1. Let $\mathrm{CYO}_{n}$, for all $n \geq 3$, where $n$ is odd, be the cyclic octahedron structure. Then, the eccentric polynomial of $\mathrm{CYO}_{n}$ is

$$
\operatorname{ECP}\left(\mathrm{CYO}_{n}, x\right)=8 n\{2 x+1\} x^{\frac{n+1}{2}}
$$

Proof. Let $\mathrm{CYO}_{n}$, where $n$ is odd, be the cyclic octahedron structure containing $5 n$ vertices and $12 n$ edges.

The formula of the eccentric polynomial is

$$
\operatorname{ECP}(G, x)=\sum_{v \in V} d_{v} x^{\varepsilon(v)}
$$

Using the vertex partition from Table 1, we obtained the following computations:

$$
\begin{gathered}
\operatorname{ECP}\left(\mathrm{CYO}_{n}, x\right)=4 \cdot 4 n \cdot(x)^{\frac{n+3}{2}}+8 \cdot n \cdot(x)^{\frac{n+1}{2}}, \\
\operatorname{ECP}\left(\operatorname{CYO}_{n}, x\right)=16 n \cdot(x)^{\frac{n+3}{2}}+8 n \cdot(x)^{\frac{n+1}{2}},
\end{gathered}
$$




$$
\operatorname{ECP}\left(\mathrm{CYO}_{n}, x\right)=8 n\{2 x+1\} x^{\frac{n+1}{2}}
$$

Theorem 2. Let $C Y O_{n}$, for all $n \geq 4$ where $n$ is even, be the cyclic octahedron structure. Then, the eccentric polynomial of $\mathrm{CYO}_{n}$ is

$$
\operatorname{ECP}\left(C Y O_{n}, x\right)=8 n\{x+2\} x^{\frac{n+2}{2}} .
$$

Proof. Let $\mathrm{CYO}_{n}$, where $n$ is even, be the cyclic octahedron structure containing $5 n$ vertices and $12 n$ edges.

The formula of the eccentric polynomial is

$$
\operatorname{ECP}(G, x)=\sum_{v \in V} d_{v} x^{\varepsilon(v)}
$$

Using the vertex partition from Table 2, we obtained the following computations:

$$
\begin{gathered}
E C P\left(\operatorname{CYO}_{n}, x\right)=8 \cdot n \cdot(x)^{\frac{n+2}{2}}+8 \cdot n \cdot(x)^{\frac{n+4}{2}}+8 \cdot n \cdot(x)^{\frac{n+2}{2}}, \\
\operatorname{ECP}\left(\mathrm{CYO}_{n}, x\right)=8 n \cdot(x)^{\frac{n+4}{2}}+16 n \cdot(x)^{\frac{n+2}{2}}, \\
\operatorname{ECP}\left(\mathrm{CYO}_{n}, x\right)=8 n\{x+2\} x^{\frac{n+2}{2}} .
\end{gathered}
$$

\subsection{Eccentric Connectivity Index}

Then, using the following theorems, we computed the eccentric connectivity index of the cyclic octahedron structure $\left(\xi\left(C Y O_{n}\right)\right)$.

Theorem 3. Let $C Y O_{n}$, for all $n \geq 3$, where $n$ is odd, be the cyclic octahedron structure. Then, the eccentric connectivity index of $\mathrm{CYO}_{n}$ is

$$
\xi\left(\mathrm{CYO}_{n}\right)=4 n\{3 n+7\} .
$$

Proof. Let $\mathrm{CYO}_{n}$, where $n$ is even, be the cyclic octahedron structure containing $5 n$ vertices and $12 n$ edges.

The formula of the eccentric connectivity index is:

$$
\xi(G)=\sum_{v \in V} d_{v} \varepsilon(v)
$$

Using the vertex partition from Table 1, we obtained the following computations:

$$
\begin{gathered}
\xi\left(\mathrm{CYO}_{n}\right)=4 \cdot 4 n \cdot\left(\frac{n+3}{2}\right)+8 \cdot n \cdot\left(\frac{n+1}{2}\right), \\
\xi\left(\mathrm{CYO}_{n}\right)=8 n \cdot(n+3)+4 n \cdot(n+1), \\
\xi\left(\mathrm{CYO}_{n}\right)=4 n\{3 n+7\} .
\end{gathered}
$$

Theorem 4. Let $\mathrm{CYO}_{n}$, for all $n \geq 4$, where $n$ is even, be the cyclic octahedron structure. Then, the eccentric connectivity index of $\mathrm{CYO}_{n}$ is

$$
\xi\left(\mathrm{CYO}_{n}\right)=4 n\{3 n+8\} .
$$


Proof. Let $C Y O_{n}$, where $n$ is even, be the cyclic octahedron structure containing $5 n$ vertices and $12 n$ edges.

The formula of the eccentric connectivity index is

$$
\xi(G)=\sum_{v \in V} d_{v} \varepsilon(v)
$$

Using the vertex partition from Table 2, we obtained the following computations:

$$
\begin{gathered}
\xi\left(\mathrm{CYO}_{n}\right)=4 \cdot(2 n) \cdot\left(\frac{n+2}{2}\right)+4 \cdot(2 n) \cdot\left(\frac{n+4}{2}\right)+8 \cdot n \cdot\left(\frac{n+2}{2}\right), \\
\xi\left(\mathrm{CYO}_{n}\right)=4 n \cdot(n+4)+8 n \cdot(n+2), \\
\xi\left(\mathrm{CYO}_{n}\right)=4 n\{3 n+8\} .
\end{gathered}
$$

\subsection{Total Eccentricity Index}

Then, using the following theorems, we computed the total eccentricity index of the cyclic octahedron structure $\left(\zeta\left(C Y O_{n}\right)\right)$.

Theorem 5. Let $\mathrm{CYO}_{n}$, for all $n \geq 3$, where $n$ is odd, be the cyclic octahedron structure. Then, the total

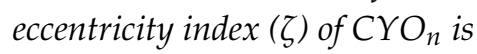

$$
\zeta\left(C Y O_{n}\right)=\frac{n}{2}\{5 n+13\}
$$

Proof. Let $\mathrm{CYO}_{n}$, where $n$ is odd, be the cyclic octahedron structure containing $5 n$ vertices and $12 n$ edges.

The formula of the total eccentricity index is

$$
\zeta(G)=\sum_{v \in V(G)} \varepsilon(v)
$$

Using the vertex partitioned from Table 3, we obtained the following computations:

$$
\begin{gathered}
\zeta\left(\mathrm{CYO}_{n}\right)=n \cdot\left(\frac{n+1}{2}\right)+4 n \cdot\left(\frac{n+3}{2}\right), \\
\zeta\left(\mathrm{CYO}_{n}\right)=\frac{n}{2}\{n+1+4 n+12\}, \\
\zeta\left(\mathrm{CYO}_{n}\right)=\frac{n}{2}\{5 n+13\} .
\end{gathered}
$$

Theorem 6. Let $\mathrm{CYO}_{n}$, for all $n \geq 4$, where $n$ is even, be the cyclic octahedron structure, then the total eccentricity index $(\zeta)$ of $\mathrm{CYO}_{n}$ is

$$
\zeta\left(\mathrm{CYO}_{n}\right)=\frac{n}{2}\{5 n+14\} .
$$

Proof. Let $\mathrm{CYO}_{n}$, where $n$ is even, be the cyclic octahedron structure containing $5 n$ vertices and $12 n$ edges.

The formula of the total eccentricity index is

$$
\zeta(G)=\sum_{v \in V(G)} \varepsilon(v) .
$$


Using the vertex partitioned from Table 4, we obtained the following computations

$$
\begin{gathered}
\zeta\left(\mathrm{CYO}_{n}\right)=3 n \cdot\left(\frac{n+2}{2}\right)+2 n \cdot\left(\frac{n+4}{2}\right), \\
\zeta\left(\mathrm{CYO}_{n}\right)=\frac{n}{2}\{3 n+6+2 n+8\} \\
\zeta\left(\mathrm{CYO}_{n}\right)=\frac{n}{2}\{5 n+14\} .
\end{gathered}
$$

\subsection{Average Eccentricity Index}

In this section, we describe how the average eccentricity index of the cyclic octahedron structure $\left(\operatorname{avec}\left(\mathrm{CYO}_{n}\right)\right)$ was determined.

Theorem 7. Let $C Y O_{n}$, for all $n \geq 3$, where $n$ is odd, be the cyclic octahedron structure. Then, the average eccentricity index $\left(\operatorname{avec}\left(\mathrm{CYO}_{n}\right)\right)$ is

$$
\operatorname{avec}\left(\operatorname{CYO}_{n}\right)=\frac{1}{10}\{5 n+13\} .
$$

Proof. Let $C Y O_{n}$, where $n$ is odd, be the cyclic octahedron structure containing $5 n$ vertices and $12 n$ edges.

The formula of the average eccentricity index is

$$
\operatorname{avec}(G)=\frac{1}{n} \sum \varepsilon_{i}
$$

Using the vertex partitioned from Table 3, we obtained the following computations:

$$
\begin{gathered}
\operatorname{avec}\left(\operatorname{CYO}_{n}\right)=\frac{1}{5 n}\left\{n \cdot\left(\frac{n+1}{2}\right)+4 n \cdot\left(\frac{n+3}{2}\right)\right\}, \\
\operatorname{avec}\left(\text { CYO }_{n}\right)=\frac{n}{10 n}\{n+1+4 n+12\} \\
\operatorname{avec}\left(\text { CYO }_{n}\right)=\frac{1}{10}\{5 n+13\} .
\end{gathered}
$$

Theorem 8. Let $\mathrm{CYO}_{n}$, for all $n \geq 4$, where $n$ is even, be the cyclic octahedron structure. Then, the average eccentricity index $\left(\operatorname{avec}\left(\mathrm{CYO}_{n}\right)\right)$ is

$$
\operatorname{avec}\left(\mathrm{CYO}_{n}\right)=\frac{1}{10}\{5 n+14\}
$$

Proof. Let $\mathrm{CYO}_{n}$, where $n$ is even, be the cyclic octahedron structure containing $5 n$ vertices and $12 n$ edges.

The formula of the average eccentricity index is

$$
\operatorname{avec}(G)=\frac{1}{n} \sum \varepsilon_{i}
$$

Using the vertex partitioned from Table 4, we obtained the following computations:

$$
\operatorname{avec}\left(\operatorname{CYO}_{n}\right)=\frac{1}{5 n}\left\{3 n \cdot\left(\frac{n+2}{2}\right)+2 n \cdot\left(\frac{n+4}{2}\right)\right\}
$$




$$
\begin{gathered}
\operatorname{avec}\left(\mathrm{CYO}_{n}\right)=\frac{n}{10 n}\{3 n+6+2 n+8\}, \\
\operatorname{avec}\left(\mathrm{CYO}_{n}\right)=\frac{1}{10}\{5 n+14\} .
\end{gathered}
$$

\subsection{First Zagreb Eccentricity Index}

In this section, we describe how we found the first Zagreb eccentricity index of the cyclic octahedron structure $\left(\mathrm{M}_{1}^{*}\left(\mathrm{CYO}_{n}\right)\right)$.

Theorem 9. Let $C Y O_{n}$ for all $n \geq 3$, where $n$ is odd, be the cyclic octahedron structure. Then, the first Zagreb eccentricity index $\mathrm{M}_{1}^{*}\left(\mathrm{CYO}_{n}\right)$ is

$$
M_{1}^{*}\left(C_{Y} O_{n}\right)=4 n\{3 n+7\} .
$$

Proof. Let $\mathrm{CYO}_{n}$, where $n$ is odd, be the cyclic octahedron structure containing $5 n$ vertices and $12 n$ edges.

The general formula of the first Zagreb eccentricity index is

$$
M_{1}^{*}(G)=\sum_{u v \in E(G)}[\varepsilon(u)+\varepsilon(v)] .
$$

By using the values from Table 5, we obtained

$$
\begin{gathered}
M_{1}^{*}\left(\mathrm{CYO}_{n}\right)=n\left(\frac{n+1}{2}+\frac{n+1}{2}\right)+6 n\left(\frac{n+1}{2}+\frac{n+3}{2}\right)+5 n\left(\frac{n+3}{2}+\frac{n+3}{2}\right), \\
M_{1}^{*}\left(\mathrm{CYO}_{n}\right)=n(n+1)+6 n(n+2)+5 n(n+3), \\
M_{1}^{*}\left(C Y O_{n}\right)=4 n\{3 n+7\} .
\end{gathered}
$$

Theorem 10. Let $\mathrm{CYO}_{n}$ for all $n \geq 4$, where $n$ is even, be the cyclic octahedron structure. Then, the first Zagreb eccentricity index $\left(\mathrm{M}_{1}^{*}\left(\mathrm{CYO}_{n}\right)\right)$ is

$$
M_{1}^{*}\left(C Y O_{n}\right)=4 n\{3 n+8\} .
$$

Proof. Let $\mathrm{CYO}_{n}$, where $n$ is even, be the cyclic octahedron structure containing $5 n$ vertices and $12 n$ edges.

The general formula of the first Zagreb eccentricity index is

$$
M_{1}^{*}(G)=\sum_{u v \in E(G)}[\varepsilon(u)+\varepsilon(v)] .
$$

By using the values from Table 6, we obtained

$$
\begin{gathered}
M_{1}^{*}\left(\mathrm{CYO}_{n}\right)=5 n\left(\frac{n+2}{2}+\frac{n+2}{2}\right)+6 n\left(\frac{n+2}{2}+\frac{n+4}{2}\right)+n\left(\frac{n+4}{2}+\frac{n+4}{2}\right), \\
M_{1}^{*}\left(\mathrm{CYO}_{n}\right)=5 n(n+2)+6 n(n+3)+n(n+4), \\
M_{1}^{*}\left(\mathrm{CYO}_{n}\right)=4 n\{3 n+8\} .
\end{gathered}
$$




\subsection{Second Zagreb Eccentricity Index}

In this section, we describe how we found the second Zagreb eccentricity index of the cyclic octahedron structure $\left(\mathrm{M}_{1}^{* *}\left(\mathrm{CYO}_{n}\right)\right)$.

Theorem 11. Let $\mathrm{CYO}_{n}$ for all $n \geq 3$, where $n$ is odd, be the cyclic octahedron structure. Then, the second Zagreb eccentricity index $\left(M_{1}^{* *}\left(C_{Y} O_{n}\right)\right)$ is

$$
M_{1}^{* *}\left(C Y O_{n}\right)=\frac{n}{4}\left\{5 n^{2}+26 n+37\right\} .
$$

Proof. Let $C Y \mathrm{O}_{n}$, where $n$ is odd, be the cyclic octahedron structure containing $5 n$ vertices and $12 n$ edges.

The general formula of the second Zagreb eccentricity index is

$$
M_{1}^{* *}(G)=\sum_{v \in V(G)}[\varepsilon(v)]^{2}
$$

By using the values from Table 3, we obtained

$$
\begin{gathered}
M_{1}^{* *}\left(\text { CYO }_{n}\right)=n\left(\frac{n+1}{2}\right)^{2}+4 n\left(\frac{n+3}{2}\right)^{2}, \\
M_{1}^{* *}\left(\text { CYO }_{n}\right)=\frac{n}{4}\left(n^{2}+2 n+1+4 n^{2}+24 n+36\right), \\
M_{1}^{* *}\left(\text { CYO }_{n}\right)=\frac{n}{4}\left\{5 n^{2}+26 n+37\right\} .
\end{gathered}
$$

Theorem 12. Let $C Y O_{n}$ for all $n \geq 4$, where $n$ is even, be the cyclic octahedron structure. Then, the second Zagreb eccentricity index $\left(\mathrm{M}_{1}^{* *}\left(\mathrm{CYO}_{n}\right)\right)$ is

$$
M_{1}^{* *}\left(\mathrm{CYO}_{n}\right)=\frac{n}{4}\left\{5 n^{2}+28 n+44\right\} .
$$

Proof. Let $\mathrm{CYO}_{n}$, where $n$ is even, be the cyclic octahedron structure containing $5 n$ vertices and $12 n$ edges.

The general formula of the second Zagreb eccentricity index is

$$
M_{1}^{* *}(G)=\sum_{v \in V(G)}[\varepsilon(v)]^{2}
$$

By using the values from Table 4, we obtained

$$
\begin{gathered}
M_{1}^{* *}\left(\mathrm{CYO}_{n}\right)=3 n\left(\frac{n+2}{2}\right)^{2}+2 n\left(\frac{n+4}{2}\right)^{2}, \\
M_{1}^{* *}\left(\mathrm{CYO}_{n}\right)=\frac{n}{4}\left(3 n^{2}+12 n+12+2 n^{2}+16 n+32\right)=\frac{n}{4}\left\{5 n^{2}+28 n+44\right\} .
\end{gathered}
$$

\subsection{Third Zagreb Eccentricity Index}

In this section we describe how we found the third Zagreb eccentricity index of the cyclic octahedron structure $\left(\mathrm{M}_{2}^{*}\left(\mathrm{CYO}_{n}\right)\right)$. 
Theorem 13. Let $C Y O_{n}$ for all $n \geq 3$, where $n$ is odd, be the cyclic octahedron structure. Then, the third Zagreb eccentricity index $\left(\mathrm{M}_{2}^{*}\left(\mathrm{CYO}_{n}\right)\right)$ is

$$
M_{2}^{*}\left(\mathrm{CYO}_{n}\right)=n\left\{3 n^{2}+14 n+16\right\} .
$$

Proof. Let $\mathrm{CYO}_{n}$, where $n$ is odd, be the cyclic octahedron structure containing $5 n$ vertices and $12 n$ edges.

The general formula of the third Zagreb eccentricity index is

$$
M_{2}^{*}(G)=\sum_{u v \in E(G)}[\varepsilon(u) \cdot \varepsilon(v)] .
$$

By using the values from Table 5, we obtained

$$
\begin{gathered}
M_{2}^{*}\left(\mathrm{CYO}_{n}\right)=n\left(\frac{n+1}{2} \cdot \frac{n+1}{2}\right)+6 n\left(\frac{n+1}{2} \cdot \frac{n+3}{2}\right)+5 n\left(\frac{n+3}{2} \cdot \frac{n+3}{2}\right), \\
M_{2}^{*}\left(\mathrm{CYO}_{n}\right)=n\left(\frac{n+1}{2}\right)^{2}+6 n\left(\frac{n^{2}+4 n+3}{4}\right)+5 n\left(\frac{n+3}{2}\right)^{2}, \\
M_{2}^{*}\left(\mathrm{CYO}_{n}\right)=n\left\{3 n^{2}+14 n+16\right\} .
\end{gathered}
$$

Theorem 14. Let $C Y O_{n}$ for all $n \geq 4$, where $n$ is even, be the cyclic octahedron structure. Then, the third Zagreb eccentricity index $\mathrm{M}_{2}^{*}\left(\mathrm{CYO}_{n}\right)$ is

$$
M_{2}^{*}\left(C Y O_{n}\right)=n\left\{3 n^{2}+16 n+21\right\}
$$

Proof. Let $\mathrm{CYO}_{n}$, where $n$ is even, be the cyclic octahedron structure contains $5 n$ vertices and $12 n$ edges.

The general formula of the third Zagreb eccentricity index is

$$
M_{2}^{*}(G)=\sum_{u v \in E(G)}[\varepsilon(u) \cdot \varepsilon(v)] .
$$

By using the values from Table 6, we obtained

$$
\begin{gathered}
M_{2}^{*}\left(\mathrm{CYO}_{n}\right)=5 n\left(\frac{n+2}{2} \cdot \frac{n+2}{2}\right)+6 n\left(\frac{n+2}{2} \cdot \frac{n+4}{2}\right)+n\left(\frac{n+4}{2} \cdot \frac{n+4}{2}\right), \\
M_{2}^{*}\left(\mathrm{CYO}_{n}\right)=5 n\left(\frac{n+2}{2}\right)^{2}+6 n\left(\frac{n^{2}+6 n+8}{4}\right)+n\left(\frac{n+4}{2}\right)^{2}, \\
M_{2}^{*}\left(\mathrm{CYO}_{n}\right)=n\left\{3 n^{2}+16 n+21\right\} .
\end{gathered}
$$

\subsection{Geometric-Arithmetic Index}

In this section, we describe how we found the eccentricity-based geometric-arithmetic index of the cyclic octahedron structure $G A_{4}\left(C Y O_{n}\right)$. 
Theorem 15. Let $\mathrm{CYO}_{n}$ for all $n \geq 3$, where $n$ is odd, be the cyclic octahedron structure. Then the geometric-arithmetic index $\left(\mathrm{GA}_{4}\left(\mathrm{CYO}_{n}\right)\right)$ is

$$
G A_{4}\left(C Y O_{n}\right)=6 n\left\{1+\frac{\sqrt{n^{2}+4 n+3}}{n+2}\right\}
$$

Proof. Let $\mathrm{CYO}_{n}$, where $n$ is odd, be the cyclic octahedron structure containing $5 n$ vertices and $12 n$ edges.

The general formula of the eccentricity-based geometric-arithmetic index is

$$
G A_{4}(G)=\sum_{u v \in E(G)} \frac{2 \sqrt{\varepsilon(u) \cdot \varepsilon(v)}}{\varepsilon(u)+\varepsilon(v)} .
$$

Using the edge partitioned from Table 5, we obtained the following computations:

$$
\begin{gathered}
G A_{4}\left(\mathrm{CYO}_{n}\right)=n\left(\frac{2 \sqrt{\frac{n+1}{2} \cdot \frac{n+1}{2}}}{\frac{n+1}{2}+\frac{n+1}{2}}\right)+6 n\left(\frac{2 \sqrt{\frac{n+1}{2} \cdot \frac{n+3}{2}}}{\frac{n+1}{2}+\frac{n+3}{2}}\right)+5 n\left(\frac{2 \sqrt{\frac{n+3}{2} \cdot \frac{n+3}{2}}}{\frac{n+3}{2}+\frac{n+3}{2}}\right), \\
G A_{4}\left(\mathrm{CYO}_{n}\right)=n\left(\frac{2 \sqrt{\left(\frac{n+1}{2}\right)^{2}}}{n+1}\right)+6 n\left(\frac{\sqrt{n^{2}+4 n+3}}{n+2}\right)+5 n\left(\frac{2 \sqrt{\left(\frac{n+3}{2}\right)^{2}}}{n+3}\right), \\
G A_{4}\left(\mathrm{CYO}_{n}\right)=6 n\left\{1+\frac{\sqrt{n^{2}+4 n+3}}{n+2}\right\} .
\end{gathered}
$$

Theorem 16. Let $\mathrm{CYO}_{n}$ for all $n \geq 4$, where $n$ is even, be the cyclic octahedron structure. Then the geometric-arithmetic index $\left(\mathrm{GA}_{4}\left(\mathrm{CYO}_{n}\right)\right)$ is

$$
G A_{4}\left(\mathrm{CYO}_{n}\right)=6 n\left\{1+\frac{\sqrt{n^{2}+6 n+8}}{n+3}\right\}
$$

Proof. Let $\mathrm{CYO}_{n}$, where $n$ is even, be the cyclic octahedron structure containing $5 n$ vertices and $12 n$ edges.

The general formula of the eccentricity-based geometric-arithmetic index is

$$
G A_{4}(G)=\sum_{u v \in E(G)} \frac{2 \sqrt{\varepsilon(u) \cdot \varepsilon(v)}}{\varepsilon(u)+\varepsilon(v)} .
$$

Using the edge partitioned from Table 6, we obtained the following computations:

$$
\begin{gathered}
G A_{4}\left(C Y O_{n}\right)=5 n\left(\frac{2 \sqrt{\frac{n+2}{2} \cdot \frac{n+2}{2}}}{\frac{n+2}{2}+\frac{n+2}{2}}\right)+6 n\left(\frac{2 \sqrt{\frac{n+2}{2} \cdot \frac{n+4}{2}}}{\frac{n+2}{2}+\frac{n+4}{2}}\right)+n\left(\frac{2 \sqrt{\frac{n+4}{2} \cdot \frac{n+4}{2}}}{\frac{n+4}{2}+\frac{n+4}{2}}\right), \\
G A_{4}\left(C Y O_{n}\right)=5 n\left(\frac{2 \sqrt{\left(\frac{n+2}{2}\right)^{2}}}{n+2}\right)+6 n\left(\frac{\sqrt{n^{2}+6 n+8}}{n+3}\right)+n\left(\frac{2 \sqrt{\left(\frac{n+4}{2}\right)^{2}}}{n+4}\right), \\
G A_{4}\left(\mathrm{CYO}_{n}\right)=6 n\left\{1+\frac{\sqrt{n^{2}+6 n+8}}{n+3}\right\} .
\end{gathered}
$$




\subsection{Atom Bond Connectivity Index}

In this section, we desscribe how we found the eccentricity-based atom bond connectivity index of the cyclic octahedron structure $\left(A B C_{5}\left(C Y O_{n}\right)\right)$.

Theorem 17. Let $C Y \mathrm{O}_{n}$ for all $n \geq 3$, where $n$ is odd, be the cyclic octahedron structure. Then, the atom bond connectivity index $\left(A B C_{5}\left(C_{Y O}\right)\right)$ is

$$
A B C_{5}\left(C Y O_{n}\right)=2 n\left\{\frac{\sqrt{n-1}}{n+1}+\frac{5 \sqrt{n+1}}{n+3}+6 \sqrt{\frac{n}{n^{2}+4 n+3}}\right\} .
$$

Proof. Let $\mathrm{CYO}_{n}$, where $n$ is odd, be the cyclic octahedron structure containing $5 n$ vertices and $12 n$ edges.

The general formula of the eccentricity-based atom bond connectivity index is

$$
A B C_{5}(G)=\sum_{u v \in E(G)} \sqrt{\frac{\varepsilon(u)+\varepsilon(v)-2}{\varepsilon(u) \cdot \varepsilon(v)}} .
$$

Using the edge partitioned from Table 5, we obtained the following computations:

$$
\begin{aligned}
& A B C_{5}\left(C_{Y} O_{n}\right)=n \sqrt{\frac{\frac{n+1}{2}+\frac{n+1}{2}-2}{\frac{n+1}{2} \cdot \frac{n+1}{2}}}+6 n \sqrt{\frac{\frac{n+1}{2}+\frac{n+3}{2}-2}{\frac{n+1}{2} \cdot \frac{n+3}{2}}}+5 n \sqrt{\frac{\frac{n+3}{2}+\frac{n+3}{2}-2}{\frac{n+3}{2} \cdot \frac{n+3}{2}}} \\
& A B C_{5}\left(\operatorname{CYO}_{n}\right)=n \sqrt{\frac{n+1-2}{\left(\frac{n+1}{2}\right)^{2}}}+12 n \sqrt{\frac{n+2-2}{n^{2}+4 n+3}}+5 n \sqrt{\frac{n+3-2}{\left(\frac{n+3}{2}\right)^{2}}}, \\
& A B C_{5}\left(C_{Y O}\right)=2 n\left\{\frac{\sqrt{n-1}}{n+1}+\frac{5 \sqrt{n+1}}{n+3}+6 \sqrt{\frac{n}{n^{2}+4 n+3}}\right\} \text {. }
\end{aligned}
$$

Theorem 18. Let $C Y O_{n}$ for all $n \geq 4$, where $n$ is even, be the cyclic octahedron structure. Then the atom bond connectivity index $\left(A B C_{5}\left(C_{Y O}\right)\right)$ is

$$
A B C_{5}\left(C_{Y O}\right)=2 n\left\{\frac{5 \sqrt{n}}{n+2}+\frac{\sqrt{n+2}}{n+4}+6 \sqrt{\frac{n+1}{n^{2}+6 n+8}}\right\} .
$$

Proof. Let $\mathrm{CYO}_{n}$, where $n$ is even, be the cyclic octahedron structure containing $5 n$ vertices and $12 n$ edges.

The general formula of the eccentricity-based atom bond connectivity index is

$$
A B C_{5}(G)=\sum_{u v \in E(G)} \sqrt{\frac{\varepsilon(u)+\varepsilon(v)-2}{\varepsilon(u) \cdot \varepsilon(v)}} .
$$

Using the edge partitioned from Table 6, we obtained the following computations:

$$
\begin{gathered}
A B C_{5}\left(C_{Y} O_{n}\right)=5 n \sqrt{\frac{\frac{n+2}{2}+\frac{n+2}{2}-2}{\frac{n+2}{2} \cdot \frac{n+2}{2}}}+6 n \sqrt{\frac{\frac{n+2}{2}+\frac{n+4}{2}-2}{\frac{n+2}{2} \cdot \frac{n+4}{2}}}+n \sqrt{\frac{\frac{n+4}{2}+\frac{n+4}{2}-2}{\frac{n+4}{2} \cdot \frac{n+4}{2}}}, \\
A B C_{5}\left(\mathrm{CYO}_{n}\right)=5 n \sqrt{\frac{n+2-2}{\left(\frac{n+2}{2}\right)^{2}}}+12 n \sqrt{\frac{n+3-2}{n^{2}+6 n+8}}+n \sqrt{\frac{n+4-2}{\left(\frac{n+4}{2}\right)^{2}}}
\end{gathered}
$$




$$
A B C_{5}\left(\mathrm{CYO}_{n}\right)=2 n\left\{\frac{5 \sqrt{n}}{n+2}+\frac{\sqrt{n+2}}{n+4}+6 \sqrt{\frac{n+1}{n^{2}+6 n+8}}\right\} .
$$

\section{Comparison}

In this section, we present tabular and graphical comparisons of the above computed indices. Table 8 shows a comparison of the eccentric connectivity index, total eccentricity, average eccentricity, first Zagreb index, second Zagreb index, third Zagreb index, atom bond connectivity index, and geometric arithmetic index for small values of $n$. Figure 3 shows a graphical comparison of the indices.

Table 8. Comparison of $\xi(G), \zeta(G)$, avec $(G), M_{1}^{*}(G), M_{1}^{* *}(G), M_{2}^{*}(G), G A_{4}(G)$, and $A B C_{5}(G)$ of $\mathrm{G} \cong \mathrm{CYO}_{n}$.

\begin{tabular}{ccccccccc}
\hline $\boldsymbol{n}$ & $\xi(G)$ & $\zeta(G)$ & $\operatorname{avec}(G)$ & $\boldsymbol{M}_{\mathbf{1}}^{*}(G)$ & $\boldsymbol{M}_{\mathbf{1}}^{* *}(G)$ & $\boldsymbol{M}_{\mathbf{2}}^{*}(G)$ & $G A_{\mathbf{4}}(G)$ & $A B C_{5}(G)$ \\
\hline 3 & 304 & 66 & 3.3 & 304 & 221 & 480 & 47.7 & 31.8 \\
4 & 320 & 68 & 3.4 & 320 & 236 & 532 & 45.8 & 31.3 \\
5 & 600 & 129 & 4.3 & 600 & 559.5 & 1248 & 71.8 & 43.7 \\
6 & 624 & 132 & 4.4 & 624 & 588 & 1350 & 70 & 43.1 \\
7 & 992 & 212 & 5.3 & 992 & 1130 & 2560 & 95.8 & 53.8 \\
8 & 1024 & 216 & 5.4 & 1024 & 1176 & 2728 & 92.5 & 53.1 \\
9 & 1480 & 315 & 6.3 & 1480 & 1992.5 & 4560 & 119.8 & 62.7 \\
10 & 1520 & 320 & 6.4 & 1520 & 2060 & 4810 & 116.2 & 62.1 \\
\hline
\end{tabular}

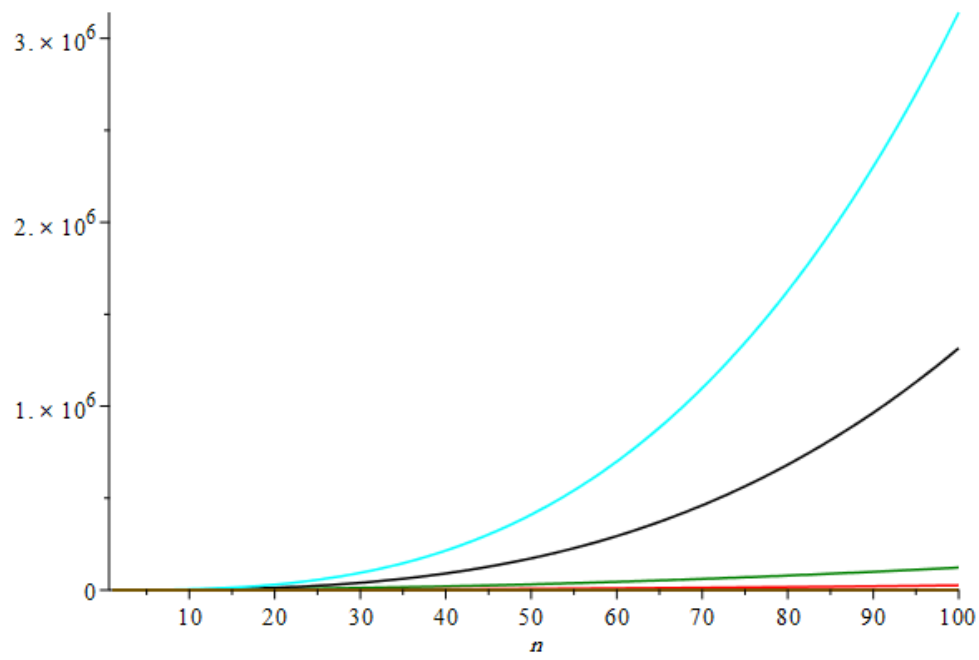

Figure 3. Graphical behavior of the eccentric indices of the cyclic octahedron structure with different colors: $\xi(G)$ is green, $\zeta(G)$ is red, $\operatorname{avec}(G)$ is blue, $M_{1}^{*}(G)=\xi(G), M_{1}^{* *}(G)$ is black, $M_{2}^{*}(G)$ is cyan, $G A_{4}(G)$ is gold, and $A B C_{5}(G)$ is orange.

\section{Conclusions}

As depicted above in Figure 3 and Table 8, one can easily see the different aspects of behavior of the cyclic octahedron structure with respect to the eccentric-based indices.

Author Contributions: The contributions of author is as; The concept, editing and supervision of the article was given by Abdul Qudair Baig. All the computations, main editing of text and use of software were made by Muhammad Naeem. Data collection and correction was made by Manzoor Ahmed Zahid. The investigation, writing first draft and methodology of the proof was done by Muhammad Razwan Azhar.

Funding: This research received no external funding.

Conflicts of Interest: The authors declare no conflict of interest. 


\section{References}

1. Gao, W.; Wang, W.; Jamil, M.K.; Farahani, M.R. Electron Energy Studying of Molecular Structures via Forgotten Topological Index Computation. J. Chem. 2016, 2016, 1053183. [CrossRef]

2. Gao, W.; Wang, W.F.; Jamil, M.K.; Farooq, R.; Farahani, M.R. Generalized atom-bond connectivity analysis of several chemical molecular graphs. Bulg. Chem. Commun. 2016, 48, 543-549.

3. Hayat, S.; Imran, M. Computation of topological indices of certain network. Appl. Math. Comput. 2014, 240, 213-228. [CrossRef]

4. Hayat, S.; Malik, M.A.; Imran, M. Computing topological indices of honeycomb derived networks. Rom. J. Inf. Sci. Technol. 2015, 18, 144-165.

5. Hayat, S.; Imran, M. Computation of certain topological indices of nanotubes covered by C5 and C7. J. Comput. Theor. Nanosci. 2015, 12, 533-541. [CrossRef]

6. Imran, M.; Hayat, S. On computation of topological indices of aztec diamonds. Sci. Int. 2014, 26, 1407-1412.

7. Baig, A.Q.; Imran, M.; Ali, H. On topological indices of poly oxide, poly silicate, DOX, and DSL networks. Can. J. Chem. 2015, 93, 730-739. [CrossRef]

8. Baig, A.Q.; Azhar, M.R.; Farahani, M.R.; Ediz, S. Some Eccentricity Based Topological Indices Of Tetra Sheets. Commun. Appl. Anal. 2017, 21,631-646.

9. Zhang, X.; Baig, A.Q.; Azhar, M.R.; Farahani, M.R.; Imran, M. The Average eccentricity and Eccentricity based Geometric-arithmetic index of tetra sheets. Int. J. Pure Appl. Math. 2017, 117, 467-479.

10. Gao, W.; Farahani, M.R.; Jamil, M.K. The eccentricity version of atom-bond connectivity index of linear polycene parallelogram benzoid $A B C_{5}(P(n, n))$. Acta Chim. Slov. 2016, 63, 376-379. [CrossRef] [PubMed]

11. Li, M.Y.; Sevov, S.C. Mellitate-based co-ordination polymers with a recurring motif: Controlling dimensionality with secondary ligands. CrystEngComm 2013, 15, 5107-5113. [CrossRef]

12. Klavzar, S.; Milutinovic, U. Graphs and a variant of the tower of Hanoi problem. Czech. Math. J. 1997, 47, 95-104. [CrossRef]

13. Hinz, A.M.; Parisse, D. The Average Eccentricity of Sierpinski Graphs. Graphs Comb. 2012, 5, 671-686. [CrossRef]

14. Ilic, A. On the extremal properties of the average eccentricity. Comput. Math. Appl. 2012, 64, $2877-2885$. [CrossRef]

15. Aouchiche, M. Comparaison Automatise D'Invariants En Theorie Des Graphes. Ph.D. Thesis, Ecole Polytechnique de Montreal, Montreal, QC, Canada, February 2006.

16. Aouchiche, M.; Bonnefoy, J.M.; Fidahoussen, A.; Caporossi, G.; Hansen, P.; Hiesse, L.; Lachere, J.; Monhait, A. Variable neighborhood search for extremal graphs, 14. The AutoGraphiX 2 System. In Global Optimization: From Theory to Implementation; Liberti, L., Maculan, N., Eds.; Springer: Berlin, Germany, 2006; pp. 281-310.

17. Caporossi, G.; Hansen, P. Variable neighborhood search for extremal graphs. I. the AutoGraphiX system. Discret. Math. 2000, 212, 29-44. [CrossRef]

18. Dankelmann, P.; Goddard, W.; Swart, C.S. The average eccentricity of a graph and its subgraphs. Util. Math. 2004, 65, 41-51.

19. Tang, Y.; Zhou, B. On average eccentricity. MATCH Commun. Math. Comput. Chem. 2012, 67, 405-423.

20. Sharma, V.; Goswami, R.; Madan, A.K. Eccentric connectivity index: A novel highly discriminating topological descriptor for structure property and structure activity studies. J. Chem. Inf. Comput. Sci. 1997, 37, 273-282. [CrossRef]

21. Alaeiyan, M.; Asadpour, J. Computing the MEC polynomial of an infinite family of the linear parallelogram $P(n, n)$, Optoelectron. Adv. Mater. Rapid Commun. 2012, 6, 191-193.

22. Alaeiyan, M.; Mojarad, R.; Asadpour, J. A new method for computing eccentric connectivity polynomial of an infinite family of linear polycene parallelogram benzenod, Optoelectron. Adv. Mater. Rapid Commun. 2011, 5, 761-763.

23. Farooq, R.; Ali Malik, M. On some eccentricity based topological indices of nanostar dendrimers. Optoelectron. Adv. Mater. Rapid Commun. 2015, 9, 842-849.

24. Bukley, F.; Harary, F. Distance in Graphs; Addison-Wesley: Redwood City, CA, USA, 1990.

25. Ghorbani, M.; Hosseinzadeh, M.A. A new version of Zagreb indices. Filomat 2012, 26, 93-100. [CrossRef]

26. Vukičević, D.; Graovac, A. Note on the comparison of the first and second normalized Zagreb eccentricity indices. Acta Chim. Slov. 2010, 57, 524-528. 
27. Ghorbani, M.; Khaki, A. A note on the fourth version of geometric-arithmetic index. Optoelectron. Adv. Mater. Rapid Commum. 2010, 4, 2212-2215.

28. Farahani, M.R. Eccentricity version of atom bond connectivity index of benzenoid family $A B C_{5}(H k)$. World Appl. Sci. J. Chem. 2013, 21, 1260-1265.

29. Perry Iv, J.J.; Perman, J.A.; Zaworotko, M.J. Design and synthesis of metal-organic frameworks using metal-organic polyhedra as supermolecular building blocks. Chem. Soc. Rev. 2009, 38, 1400-1417. [CrossRef] [PubMed]

(C) 2018 by the authors. Licensee MDPI, Basel, Switzerland. This article is an open access article distributed under the terms and conditions of the Creative Commons Attribution (CC BY) license (http:/ / creativecommons.org/licenses/by/4.0/). 